\title{
A characterization of some additive arithmetical functions, III
}

\author{
by \\ Jean-Loup Mauclaire (Paris)
}

I. Introduction. In 1946, P. Erdős [2] proved that if a real-valued additive arithmetical function $f$ satisfies the condition: $f(n+1)-f(n) \rightarrow 0$, $n \rightarrow \infty$, then there exists a constant $C$ such that $f(n)=C \log n$ for all $n$ in $\mathbb{N}^{*}$. Later, I. Kátai $[3,4]$ was led to conjecture that it was possible to determine additive arithmetical functions $f$ and $g$ satisfying the condition: there exist a real number $l, a, c$ in $\mathbb{N}^{*}$, and integers $b, d$ such that $f(a n+b)-g(c n+d) \rightarrow l, n \rightarrow \infty$. This problem has been treated essentially by analytic methods ([1], [7]). In this article, we shall provide, in an elementary way, a characterization of real-valued additive arithmetical functions $f$ and $g$ satisfying the condition:

(H) there exist $a$ and $b$ in $\mathbb{N}^{*}$ with $(a, b)=1$ and a finite set $\Omega$ such that

$$
\lim _{n \rightarrow \infty} \min _{\omega \in \Omega}|f(a n+b)-g(n)-\omega|=0 .
$$

II. Results. We have the following result:

THEOREM. Let $f$ and $g$ be real-valued additive arithmetical functions satisfying the condition $(\mathrm{H})$. Then there exists a constant $C$ such that the set of values of the sequences $g(n)-C \log n, n$ in $\mathbb{N}^{*}$, and $f(n)-C \log n$, $(n, a)=1$, is finite.

Acknowledgments. The author thanks the referee who provided a real simplification of the proof given in the first version of this article, and some nice cosmetical modifications to the original text.

III. Proof of the Theorem. We shall always assume that $f\left(p^{k}\right)=0$ for all primes $p$ dividing $a$ and all $k$, since a change of these values does not affect the assumption of the Theorem.

1991 Mathematics Subject Classification: Primary 11N64. 
The proof relies essentially on the following result which can be obtained elementarily:

THEOREM A. Let $f$ and $g$ be real-valued additive arithmetical functions satisfying the condition: there exist $a$ and $b$ in $\mathbb{N}^{*}$ with $(a, b)=1$ such that $f(a n+b)-g(n)=O(1)$. Then there exists a constant $C$ such that $g(n)-C \log n$ and $f(n)-C \log n$ remain bounded.

Pr o o f. For an announcement of this result, see [6], and for a proof, [5].

ASSERTION 1. Let $f$ and $g$ satisfy the hypothesis $(\mathrm{H})$. Then there exist a constant $C$ and bounded additive arithmetical functions $g^{\prime}$ and $f^{\prime}$ such that $f(n)=C \log n+f^{\prime}(n), g(n)=C \log n+g^{\prime}(n)$.

Proof. By our hypothesis, there exists a finite set $\Omega$ such that

$$
\lim _{n \rightarrow \infty} \min _{\omega \in \Omega}|f(a n+b)-g(n)-\omega|=0 .
$$

This gives us that $f(a n+b)-g(n)$ is bounded, and Assertion 1 is an immediate consequence of Theorem A.

We only have to prove

Proposition. Let $f$ and $g$ be bounded real-valued additive arithmetical functions satisfying the condition $(\mathrm{H})$. Then the set of values of $f(n)$ and $g(n)$ is finite.

To prove this, we introduce the functions $f_{p}(n)=f\left(p^{k}\right)$ if $p^{k} \| n$ for each prime $p$, and similarly $g_{p}$. Let $V_{p}$ denote the set of values of the function $f_{p}(a n+b)-g_{p}(n)$. The next assertion is trivial:

Assertion 2. An additive function $h(n)$ is bounded if and only if

$$
\sum_{p} \max _{k}\left|h\left(p^{k}\right)\right|<\infty .
$$

Main Lemma. Let $q \geq 2$ and $v$ be any element of the set $\sum_{p \leq q} V_{p}$. Then $v$ is a limit point of $f(a n+b)-g(n)$.

Proof. We have $v=\sum_{p \leq q} v_{p}$ with $v_{p}=f_{p}\left(a n_{p}+b\right)-g_{p}\left(n_{p}\right)$. For every $n$ we have

$$
f(a n+b)-g(n)=\sum_{p}\left(f_{p}(a n+b)-g_{p}(n)\right) .
$$

We want to find infinitely many values of $n$ for which this is in $(v-\varepsilon, v+\varepsilon)$. To achieve this, it is sufficient that

$$
f_{p}(a n+b)-g_{p}(n)=f_{p}\left(a n_{p}+b\right)-g_{p}\left(n_{p}\right)
$$

for all $p \leq q$, and

$$
\sum_{p>q}\left|f_{p}(a n+b)-g_{p}(n)\right|<\varepsilon
$$


For (3), it is sufficient that $n$ contains $p$ with the same exponent as $n_{p}$, and $a n+b$ with the same exponent as $a n_{p}+b$. Both are satisfied if

$$
n \equiv n_{p}\left(\bmod p^{k_{p}+1}\right),
$$

where $k_{p}$ is the exponent of $p$ in $n_{p}\left(a n_{p}+b\right)$.

To treat (4), we first select a $Q$ so that

$$
\sum_{p>Q} \max _{k}\left|f\left(p^{k}\right)\right|+\max _{k}\left|g\left(p^{k}\right)\right|<\varepsilon
$$

(Assertion 2). Then the contribution of primes $p>Q$ in (4) is $<\varepsilon$. Thus (4) will hold if we achieve that

$$
f_{p}(a n+b)-g_{p}(n)=0
$$

for all $q<p<Q$. To do this, it is sufficient that $p \nmid n(a n+b)$. Since this excludes two residue classes for $p \nmid a$ and one for $p \mid a$, we can find $b_{p}$ such that the assumption

$$
n \equiv b_{p}(\bmod p)
$$

guarantees (6).

Hence every $n$ that satisfies the congruence (5) for all $p \leq q$ and (7) for all $q<p \leq Q$ also satisfies $|f(a n+b)-g(n)-v|<\varepsilon$. There are infinitely many such numbers, and consequently $v$ is a limit point.

End of proof of the Proposition. We know that the sumset $\sum_{p \leq q} V_{p}$ is contained in $\Omega$ for all $q$; so the cardinalities of these sets are bounded. This means that each $V_{p}$ is finite, and with a finite number of exceptions $\left|V_{p}\right|=1$.

Write

$$
F_{p}=\left\{0, f(p), f\left(p^{2}\right), \ldots\right\}, \quad G_{p}=\left\{0, g(p), g\left(p^{2}\right), \ldots\right\} .
$$

The property that $f$ and $g$ have only a finite number of values is equivalent to saying that $F_{p}$ and $G_{p}$ are always finite and are equal to $\{0\}$ except for finitely many primes. For most primes this will follow from the lemma below.

Lemma. For $p \nmid 2 a b$ we have $V_{p}=F_{p} \cup\left(-G_{p}\right)$.

Proof. If $p \nmid b$, then we cannot have both $p \mid n$ and $p \mid a n+b$, thus $V_{p} \subset$ $F_{p} \cup\left(-G_{p}\right)$ follows. To show the other inclusion, for a given $k \geq 1$ take an $n$ such that $p^{k} \| n$; then $p \nmid a n+b$ and so

$$
f_{p}(a n+b)-g_{p}(n)=-g_{p}(n)=-g\left(p^{k}\right) .
$$

Similarly, as $p \nmid a$ we can find an $n$ with $p^{k} \| a n+b$ and we infer $f\left(p^{k}\right) \in V_{p}$. Finally, since $p>2$ we can find an $n$ with $p \nmid n(a n+b)$ to show $0 \in V_{p}$.

For $p \mid 2 a b$ a complete description of $V_{p}$ would split into several subcases, but for our aims the following weaker assumption suffices.

Lemma. If $V_{p}$ is finite, so are $F_{p}$ and $G_{p}$. 
Proof. Write $p^{r} \| b$. By considering $n=p^{k}$ we see that $f\left(p^{r}\right)-g\left(p^{k}\right) \in V_{p}$ for $k>r$, thus $g\left(p^{k}\right) \in-V_{p}+f\left(p^{r}\right)$ and $G_{p}$ is finite.

If $p \mid a$, then $F_{p}=\{0\}$ by assumption. If $p \nmid a$, then we can find $n$ such that $p^{k} \| a n+b$ and we obtain $f\left(p^{k}\right)-g\left(p^{r}\right) \in V_{p}, f\left(p^{k}\right) \in V_{p}+g\left(p^{r}\right)$.

These lemmas show that $F_{p}, G_{p}$ are always finite, and they are $\{0\}$ whenever $p \nmid 2 a b$ and $\left|V_{p}\right|=1$, hence for all but finitely many $p$. This concludes the proof of the Theorem.

\section{References}

[1] P. D. T. A. Elliott, The value distribution of differences of additive arithmetic functions, J. Number Theory 32 (1989), 339-370.

[2] P. Erdös, On the distribution function of additive functions, Ann. of Math. 47 (1946), 1-20.

[3] I. Kátai, Some results and problems in the theory of additive functions, Acta Sci. Math. (Szeged) 30 (1969), 305-311.

[4] -, On number theoretical functions, in: Number Theory (Colloq., János Bolyai Math. Soc., Debrecen, 1968), North-Holland, Amsterdam, 1970, 133-137.

[5] J. L. Mauclaire, Contributions à la théorie des fonctions additives, thèse, Publ. Math. Orsay 215, 1977.

[6] —, Sur la régularité des fonctions additives, Sém. Delange-Pisot-Poitou (15 ième année, 1973/1974), fasc. 1, exp. no. 23, 1975.

[7] N. M. Timofeev, Integral limit theorems for sums of additive functions with shifted arguments, Izv. Math. 59 (1995), 401-426.

Institut de Mathématiques

U.M.R. 7586 du C.N.R.S.

Case 247

4 Place Jussieu

75252 Paris Cedex 05, France

E-mail: mauclai@ccr.jussieu.fr 